\title{
Disertaciones elementales sobre teoría combinatoria: hacia una inculturación de las matemáticas
}

Marcos Antonio Reyes Centeno ${ }^{1}$

\section{Resumen}

En este trabajo se abordó la enseñanza de la teoría combinatoria con dos tipos de problemas, aquellos cuyos resultados pueden ser comprobados por conteo directo y los que no pueden verificarse, porque la cantidad de eventos obtenidos no permiten por cuestiones de tiempo, verificar uno a uno, todos los arreglos obtenidos. Además se determinó la utilidad didáctica y académica de ambos tipos de problemas. Otro aspecto que se incluyó es la atención a las singularidades, como parte de la atención a la diversidad: La atención particularizada que ha de darse a los estudiantes de avanzada.

Se incorporó en este trabajo, una serie de ejemplos sobre problemas conmensurables y su uso didáctico, que no pretenden, por la brevedad del documento, dar una respuesta definitiva sobre la enseñanza de las técnicas de conteo, si no mostrar levemente, los posibles derroteros para abordar didácticamente esta apasionante temática. Se visualizaron también ejemplos de problemas inconmensurables y su utilidad académica, para atender las singularidades de estudiantes amantes de las matemáticas.

Palabras clave: Atención a la diversidad; teoría combinatoria; contextualización.

\section{Summary}

In this work, we focused on the teaching of combinatorial theory with two types of problems: those which the results can be verified by direct counting and those that cannot be verified, because the number of events do not allow verifying one by one all the arrangements obtained due to time issues. Besides, we determined the didactic and academic utility of both types of problems. Another aspect that was included is the attention to singularities, as part of the attention to diversity: meaning the particular attention that must be given to advanced students.

A series of examples on commensurable problems and its didactic use were incorporated, which don't pretend -due to time- to give a definitive answer on the teaching of counting techniques, but rather to show slightly the possible ways to address this

1 Candidato a Doctor en Educación e Intervención Social por la Universidad Pablo Olavide, Vice-Decano de la Facultad Regional Multidisciplinaria de la UNAN-Managua, con sede en Chontales. Correo electrónico: mreyescent@gmail.com 
exciting theme in a didactic manner. Examples of incommensurable problems and its academic utility were also visualized in order to attend the singularities of students who love mathematics.

Keywords: Attention to diversity; combinatorial theory; contextualization.

\section{Introducción}

El análisis combinatorio o como suele llamarse: Técnicas de conteo, es una temática que presta condiciones para el desarrollo de actividades educativas innovadoras dentro del aula de clase y fuera de ella, debido a que los algoritmos y las fórmulas que se establecen en esta interesante área de las matemáticas, tienen un papel muy utilitario y no son el asunto central para el desarrollo de los procesos de comprensión y análisis que comportan los problemas que se abordan en este contenido.

Hay que reconocer, no obstante que en el abordaje de los tópicos de teoría combinatoria, se pueden establecer dos tipos de problemas, con dos tipos de dimensiones: Los problemas básicos que se pueden trabajar en el aula de clase y cuyos resultados son fácilmente observables, medibles y responden a la dimensión conmensurable y aquellos problemas de un estricto orden académico, que responden a variados, contextos universales, cuyos resultados no son fácilmente observables y que tienen una dimensión inconmensurable, al menos en los límites de tiempo que los estudiantes tienen establecidos para superar las asignaturas y promover.

Desde esta perspectiva, en esta disertación, se abordan los dos tipos de problemas, con sus dimensiones particulares, especial énfasis en los problemas que pueden ser analizados con situaciones del contexto, que de seguro han de garantizar aprendizajes más significativos, que el segundo tipo de problemas de esta temática.

El trabajar situaciones contextuales desde cualquier tema de matemáticas, debe ser una necesidad, porque el aprendizaje que perdura, es aquel que se puede asociar con las vivencias particulares del discente y no el que está determinado por las capacidades académicas e intelectuales del docente.

No obstante, no se puede descuidar el hecho de la atención a la diversidad y, en este sentido, en esta arenga, se aboga, por la atención a las particularidades de los jóvenes o estudiantes de avanzada. ¿Y por qué esto? porque por lo general, la búsqueda de la atención a la diversidad, está orientada a la adecuación, tanto académica como metodológica, de los contenidos para los diversos estilos de aprendizaje, que en la práctica, lo que hace es establecer estándares mínimos de aprendizajes, y con eso, se atiende de modo concreto la diversidad. 
Esto es bueno, en parte, no obstante pocas veces se atiende la singularidad de los estudiantes, que aprenden con mayor ritmo y que requieren enfrentar situaciones, que le planteen retos y que los impulsen a hacer esfuerzos intelectuales de muy buen nivel. Si en todos los estudiantes, se provoca el desarrollo de unas competencias fundamentales, esto conduce a una estandarización, una normalización platicúrtica, sin los apuntalamientos adecuados y prudentes que tomen en cuenta a aquellos individuos, que por sus características naturales, se esfuerzan más que los demás y aprenden más rápido y con más eficiencia que el resto.

En este caso, hablamos de estudiantes a los que se les puede aplicar una de las máximas de la filosofía Gassetiana:

El hombre selecto no es el petulante que se cree superior a los demás, sino el que se exige más que los demás, aunque no logre cumplir en su persona esas exigencias superiores." (Ortega y Gasset, 1926, pág. 26)

Cuando nos referimos a estos tipos de estudiantes singulares, debemos entender que son aquellos que buscan el desarrollo de su talento al tope de sus posibilidades, aunque no lo consigan en un $100 \%$. Al trabajar con esta particularidad de discentes, debemos tener clara la idea de actuar en pro de ellos y con la visión de formarlos con más y mejores niveles académicos, sin descuidar la formación de su humildad y su humanidad.

\section{Desarrollo}

En cierto sentido la teoría combinatoria puede considerase tan vieja como la propia matemática, ya que la operación básica de contar los elementos de un conjunto está ligada al origen mismo del concepto de número en los tiempos prehistóricos.

Los matemáticos griegos no prestaron mucha atención a los problemas combinatorios, si exceptuamos del estudio de los números poligonales realizados por los pitagóricos.

Cardano demostró que el número de subconjuntos de un conjunto de n elementos, es $2^{n}$.

Tartaglia estudió un rectángulo aritmético equivalente al triángulo que posteriormente recibiría el nombre de Pascal.

Pascal parece haber sido el primero en relacionar los números $\left(\begin{array}{l}n \\ r\end{array}\right)$ con el teorema del binomio (el cual en alguna forma ya era conocido por los árabes en el siglo XIII y por los chinos en el siglo XIV) (Nieto, 1996, págs. 2-3). 
Leibniz (1616-1716) dedicó bastante atención a la combinatoria no sólo desde el punto de vista matemático sino también desde una perspectiva filosófica. En un ensayo de juventud ("De Arte Combinatoria, 1666") escribe:

... todo brota interiormente de la teoría de las variaciones la cual conduce al espíritu que a ella se confía, casi por sí mismo, a través de la totalidad infinita de los problemas, abarcando en sí la armonía del universo, la estructura más íntima de las cosas y toda la serie de las formas (Nieto, 1996, pág. 3).

En la actualidad la teoría combinatoria, es necesaria para el cálculo de probabilidades, la teoría de grafos, las funciones de Möbius, etc.

\section{¿Cómo abordar la teoría combinatoria, en estas aulas de clase?}

En los programas del Ministerio de Educación de Nicaragua, se aborda la teoría combinatoria, particularmente, se hace especial énfasis, en el principio fundamental de conteo, las combinaciones y las permutaciones. La teoría combinatoria, debe abordarse de una forma asociada a cosas que el estudiante pueda ver en el día a día, esto es el principio de la contextualización de los aprendizajes.

Es recomendable para la enseñanza de esta temática, iniciar con situaciones de orden combinatorio que puedan ser observadas en la realidad y cuyos resultados, sean visibles y medibles, estamos hablando de la dimensión conmensurable en la enseñanza de este contenido.

Se propone, en este trabajo algunas formas en que se pueden abordar algunos tópicos particulares de la Escuela Secundaria asociados o relacionados con este tema.

Ejemplos contextuales.

Ej. 1: Tú tienes tres camisas y dos pantalones ¿Cuántas formas distintas tienes para vestirte usando esas prendas?

- Ahora supón que tienes tres camisas y cinco pantalones. ¿Cuántas formas distintas de vestirte tienes?

- Si tu hermano te pide prestado un pantalón ¿De cuántas maneras puedes vestirte ahora?

- ¿Disminuyen o aumentan tus alternativas? ¿Cuál es el factor de disminución.

- Explica a qué se debe esa disminución o ese aumento.

Para el desarrollo de esta actividad, es necesaria la utilización de materiales concretos para resolver el asunto inicial, que debe conllevar al estudiante a deducir que el número de formas en que puede vestirse con dos pantalones y tres camisas, 
está dado por el producto: $2 \times 3=6$ maneras distintas de combinar la ropa. Por tanto, hasta que hayan descubierto la operación implícita después de haber realizado todas las combinaciones posibles, con recortes de camisa y pantalones distintos, que el docente debe facilitar de previo, se debe decir, que a esta técnica se le llama principio fundamental de conteo. Es necesario que a los estudiantes se les dé la oportunidad de agotar todo su cúmulo de ideas, y el maestro no debe intervenir hasta que la discusión y el análisis de los estudiantes esté en un punto álgido, si fuese el caso.

\section{Principio de la multiplicación en el conteo}

Llamado también, "principio fundamental del análisis combinatorio" y puede enunciarse como sigue: Si una cosa puede hacerse de " $n_{1}$ " formas diferentes, una segunda cosa puede hacerse de " $n_{2}$ " formas diferentes, una tercera cosa puede hacerse de " $n_{3}$ " formas diferentes, etc., una r-ésima cosa puede hacerse de $\mathrm{n}_{\mathrm{r}}$ formas diferentes, todo el conjunto puede hacerse de: $n_{1} \cdot n_{2} \cdot n_{3} \cdot \ldots \cdot n_{r}$ (Gómez, 2009, pág. 76)

- Hay que aprovechar, también, el momento para introducir problemas de conteo que no presentan estructura multiplicativa. Esto debe verse como una excepcionalidad.

- No todos los problemas de conteo son abordables desde la óptica de las estructuras multiplicativas.

A continuación se presenta un problema que no se resuelve por el principio de la multiplicación, pues no se aplica éste; para que el alumno discrimine entre un tipo de problema y otro, pero en ambos se necesita contar.

Ej. 1: ¿De cuántas formas se pueden repartir 36 caramelos en paquetes iguales sin que sobre ninguno?

Se pueden repartir en tantos grupos como divisores tiene el 36, de tal modo que el número de formas es equivalente al número de divisores. (Estos podemos calcularlos, sabiendo que: $36=2^{2} \times 3^{2}$ y que el número de divisores es $(2+1)(2+1)=9$, como se muestra a continuación.

$D=(36,18,9,12,6,4,3,2,1)$

Siempre bajo la idea de presentar los contenidos, concatenados con situaciones de fácil manejo y comprensión, aprovechando además, las ventajas que ofrecen los artículos tecnológicos que están en manos de los estudiantes, se plantea el siguiente problema de permutaciones, utilizando el celular como cámara fotográfica.

Esta actividad se realizará bajo la conducción del profesor, con apoyo de tres estudiantes. 
Ej. 3: Los estudiantes ubicarán tres sillas delante la clase y se pedirá la participación de tres de ellos (las ordenaciones se harán anotando los nombres de los alumnos) para realizar una sesión fotográfica, luego se cambiará la posición de los alumnos en las sillas hasta agotar las posibles distribuciones. Un estudiante anotará los resultados posibles.

\section{Se preguntará:}

- ¿Qué pudimos observar? ¿Y si se colocaran 4 sillas con 4 estudiantes?

- ¿De cuántas formas podrían acomodarse los estudiantes para otra sesión fotográfica? (estos resultados sólo se anotarán en la pizarra y por escrito en cuadernos de trabajo).

- ¿Cuántas veces está contenido el primer resultado en éste?

Y si fueran cinco estudiantes en cinco sillas ¿Cuántas veces está contenido el segundo resultado en éste?

\section{¿Qué concluyes?}

Es posible que para un estudiante que está acostumbrado a la enseñanza tradicional, los retos que presenta la verbalización de los conocimientos o al menos de las ideas que tienen sobre el problema en cuestión, sean una traba al inicio, pero en la medida que este tipo de actividades sea común en los procesos de enseñanza aprendizaje, el análisis y la comunicación de estas experiencias cognitivas, serán cada vez más comunes y de fácil ejecución para la mayoría de los estudiantes.

En ninguna de las situaciones críticas en esta primera parte del trabajo, se plantea cómo han de hacerse los procesos para la consecución de las soluciones, es decir no se plantea la resolución de los problemas, por ser éstos de un nivel muy elemental, puesto que a partir del primero, se debe de obtener el patrón para conseguir las resoluciones de los otros, lógicamente con la ayuda del papel mediador del docente, que ha de procurar no interferir mucho para no influir en el razonamiento de los estudiantes.

\section{Notación de factorial}

El producto de los enteros positivos desde el uno hasta " $n$ " inclusive, se emplea con mucha frecuencia en matemáticas y aquí lo denotamos por el símbolo especial $n !$, tal que $n !=1 \cdot 2 \cdot 3 \cdot 4 \cdots(n-4) \cdot(n-3) \cdot(n-2) \cdot(n-1) \cdot n$ (Lipschutz, 1997, pág. 16).

He aquí otro problema, de orden muy contextual, pero que requiere un alto espíritu de reflexión y pensamiento crítico por parte de los estudiantes. 
Ej. 4: Formando grupos de 4 estudiantes, se les orientará que usando la calculadora científica, específicamente la tecla de factorial, se asignará a cada estudiante una de las cuatro actividades que a continuación se presentan, pasando al frente cuatro estudiantes seleccionados para presentar reflexiones.

- ¿A partir de qué número los factoriales son divisibles por tres? ¿Por qué?

- ¿A partir de qué números los factoriales terminan en un cero? ¿Por qué?

- ¿A partir de qué números los factoriales terminan en dos ceros? ¿Y Por qué?

- ¿Cuál es el mayor número al que se le puede calcular el factorial en una calculadora científica? ¿Por qué?

Aunque en esta actividad, los estudiantes recurrirán de inicio al método del ensayo y error, las preguntas en las que se piden las razones para que cada evento ocurra, obliga al estudiante a activar los mecanismos mentales, para dar las respuestas. Se insiste siempre, en que la participación del docente para conducir adecuadamente el proceso, ha de ser relegada a un segundo plano y debe estar condicionada a las dificultades presentadas en la resolución de la tarea por parte de los estudiantes.

Todas las situaciones presentadas anteriormente, tienen elementos en común: El planteamiento inicial permite al estudiante, poder verificar el número de resultados posibles que demanda cada situación o permitir hacer los ensayos necesarios en el tiempo. El nivel de complejidad para el análisis de problemas es básico y no requiere la estructuración de resoluciones ingeniosas. Se busca el desarrollo del pensamiento crítico y la reflexión a través de preguntas estructuradas para tal fin.

\section{Problemas con un enfoque más académico}

Esta parte de este trabajo, muestra la forma de resolver algunas situaciones de teoría combinatoria, que tienen un mayor grado de complejidad para las que se debe de hacer uso del pensamiento estructurado o convencional más una dosis extra de pensamiento lateral, al que algunos profesores de matemáticas suelen, llamar el tercer ojo matemático, en alusión al bindi hindú. Esto se aplica para los casos en que al resolver problemas, algunas personas ven aspectos que otros no ven.

En este caso los procesos intuitivos más el razonamiento abstracto, son necesarios y fundamentales para comprender los problemas que se presentan.

Ej.1 ¿De cuántas maneras 3 españoles, 4 franceses y 2 italianos, se pueden ubicar en una fila, si los de la misma nacionalidad, deben quedar juntos?

Éste un problema de análisis combinatorio, no presenta gran dificultad. Tal vez la dificultad estribe, en que no sea muy fácil verificar la cantidad de arreglos que el problema implica. 


\section{EDUCACIÓN}

- Aquí se puede utilizar como estrategia, cambiar de campo el problema, en este caso mirarlo en chiquito para estudiar su comportamiento.

- ¿De cuántas formas se pueden ubicar en una fila, dos españoles y dos franceses, si los de la misma nacionalidad, deben quedar juntos?

Aquí limitamos el problema, de tal modo que los arreglos posibles se puedan "contar" en poco tiempo. Es importante observar que este nuevo problema tiene la estructura del problema original. Estudiemos su comportamiento.

\begin{tabular}{llll|llll} 
E1 & E2 & F1 & F2 & F1 & F2 & E1 & E2 \\
E1 & E2 & F2 & F1 & F1 & F2 & E2 & E1 \\
E2 & E1 & F1 & F2 & F2 & F1 & E1 & E2 \\
E2 & E1 & F2 & F1 & F2 & F1 & E2 & E1
\end{tabular}

Como se puede observar hay ocho resultados, de tal modo que los dos de cada nacionalidad se permutan dentro del mismo bloque, pero también permutan los bloques, puesto que en la primera imagen están primero los españoles y en la segunda están de primero los franceses, entonces el resultado es: 2 !x2!x2!=8 formas posibles de ubicarlos en la fila.

Continuando con este patrón, en el problema original, el resultado sería el siguiente: 3 !x4!x2!x3!=1728 maneras.

Aquí se hace lo mismo, se analizan las formas en que se permutan los de la misma nacionalidad en cada bloque y luego cómo se permutan los bloques, como son tres nacionalidades, se permutan de 3 ! maneras.

Ej. 2 Si no se permiten repeticiones $A$ ) ¿cuántos números de tres dígitos se pueden formar con los dígitos 2, 3, 5, 6, 7 y 9? B) ¿cuántos de éstos son menores que 400? C) ¿cuántos son pares? D) ¿cuántos son impares? E) ¿cuántos son múltiplos de cinco?

Utilizaremos, como estrategia, el uso de casillas, de la manera siguiente:

Para cada caso, ha de considerarse que hay que rellenar tres casillas y para ello, se cuenta con seis dígitos de los que hay que elegir tres (no hay que perder de vista que no se permiten dígitos repetidos)
a)

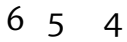

Tenemos seis opciones para rellenar la primera casilla, cinco para la segunda casilla y cuatro para la tercera. Por el principio de la multiplicación, tenemos: $6 \times 5 \times 4=$ 120 números de tres dígitos. 


\section{b)}

254

Para que bajo estas condiciones, un número de tres dígitos sea menor que 400, ha de comenzar con el dígito 2 o con el dígito 3, si así es, no importa el valor de los otros dos dígitos. Sólo mantenemos firme la idea, de que no puede haber repetición. Por tanto, tenemos dos opciones para rellenar la primera casilla, cinco para la segunda

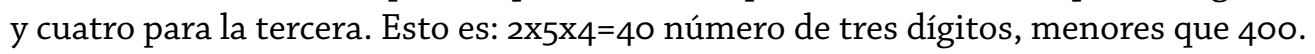

c)

$\begin{array}{lll}4 & 5 & 2\end{array}$

Para trabajar esta situación, en la que se pide, que los números de tres dígitos obtenidos han de ser pares, hay que tener en cuenta, que un número es par si termina en un número par o en cero, por tanto la última casilla, hay que considerar que ha de ser rellenada, sólo por los números pares de la serie de números que da el problema. A saber, son dos los números pares: 2 y 6, por tanto tenemos dos opciones, para rellenar la última casilla, en las dos restantes, se pueden utilizar cualquiera de los otros números, una vez que se ha elegido uno. Esto es: $4 \times 5 \times 2=40$ números pares.

d)

$\begin{array}{lll}4 & 5 & 4\end{array}$

Aquí hay dos posibilidades para resolver esta situación, sabemos que cualquier número natural, es par o es impar (el conjunto de números pares es complemento del conjunto de números impares y esta relación es biunívoca). Por tanto, para resolver este caso, basta tomar el total de números de tres dígitos que se pueden formar teniendo en cuenta las condiciones del problema y restarle la cantidad de números pares que ya se calculó, esto es: $120-40=80$ números impares.

La otra opción es, hacer lo mismo que se hizo para calcular los números pares: tenemos 4 opciones para rellenar la última casilla, cinco para la penúltima y 4 opciones para la antepenúltima, esto es: $4 \times 5 \times 4=80$ números impares.

e)

$\begin{array}{lll}4 & 5 & 1\end{array}$

Sabemos que para que un número sea múltiplo de cinco, éste debe terminar en cero o en cinco; como en la serie de números del problema no está presente el cero, 
el conjunto de números de tres cifras que debe calcularse ha de terminar en cinco, pensando en que en la última casilla, debe ir el cinco, las dos casillas restantes pueden ser ocupadas por cualquiera de los números de la serie que quedan. Tenemos entonces: 1X5×4=20 números divisibles por cinco.

EJ. 3 Se escogen aleatoriamente uno tras otro, 3 personas entre 10 hombres y 5 mujeres. Cuántas formas diferentes hay, de tal forma que: a) El primero y el tercero sean del mismo sexo y el segundo del sexo contrario. b) queden seleccionados $2 \mathrm{mu}-$ jeres y un hombre, pero las mujeres queden seguidas.

a) Para este caso hay que considerar dos situaciones: 1. Que la primera persona y la tercera persona, sean hombres.2. Que la primera y la tercera persona, sean mujeres.

Se ilustra la situación a continuación:

$\begin{array}{llllll}10 & 5 & 9 & 5 & 10 & 4\end{array}$

Resolviendo nos queda lo siguiente: $10 \times 5 \times 9+5 \times 10 \times 4=650$ ordenaciones.

b) En esta situación hay dos posibilidades: que las dos mujeres sean seleccionadas, consecutivamente, de primero o que las dos mujeres sean seleccionadas, consecutivamente, de último; esto es:

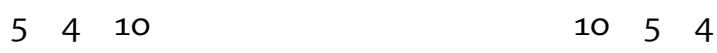

De tal modo que nos queda: $5 \times 4 \times 10+10 \times 5 \times 4=400$ arreglos posibles.

\section{Conclusión}

El abordaje de la teoría combinatoria en la Escuela Secundaria puede adquirir mucha relevancia, si se utiliza como estrategia la contextualización de los aprendizajes, para hacer esta temática más visible; para ello es necesario que el estudiante sienta que lo que está aprendiendo, tiene sentido, tiene significado y que puede ser incorporado a situaciones del diario vivir, sin menoscabo de la inclusión de tareas que fuercen al límite el pensamiento de los que son más apasionados por la ciencia de los números y los símbolos. Es decir atender la diversidad, pero en toda su extensión. La diversidad bien entendida debe respetar los ritmos de aprendizaje y los intereses de los estudiantes menos avanzados, sin descuidar las peculiaridades de los más avanzados.

Es por ello que en este trabajo, muy breve por cierto, se abordan problemas de orden contextual y otros con cierto rigor académico. En teoría los primeros, deben de ser más holísticos y los segundos deben de estar orientados a "divertir" a los jóvenes peculiares con mayor nivel de desarrollo. 
Este breve ensayo ha sido pensado, particularmente, para docentes que trabajan en Educación Secundaria y por qué no para los que trabajamos en las universidades y que debemos de entender también la diversidad en todas sus dimensiones, aun cuando enseñamos teoría combinatoria o cualquier otro tema de matemáticas.

\section{Lista de referencias}

Gómez, H. (2009). Estadística. Manizales: No indicada.

Lipschutz, S. (1997). Probabilidad. México: Mcgraw Hill.

Nieto, J. (1996). Teoría Combinatoria. Zulia: Luz.

Ortega y Gasset, J. (1926). La rebelión de las masas. Madrid: PSIKOLIBRO. 